PROCEEDINGS OF THE AMERICAN MATHEMATICAL SOCIETY

Volume 124, Number 8, August 1996

\title{
NON-TRIVIAL HARMONIC SPINORS ON GENERIC ALGEBRAIC SURFACES
}

\author{
D. KOTSCHICK
}

(Communicated by Ronald J. Stern)

\begin{abstract}
We show that there are simply connected spin algebraic surfaces for which all complex structures in certain components of the moduli space admit more harmonic spinors than predicted by the index theorem (or RiemannRoch). The dimension of the space of harmonic spinors can exceed the absolute value of the index by an arbitrarily large number.
\end{abstract}

For every closed Riemannian spin manifold its Dirac operator is a selfadjoint linear elliptic operator acting on sections of the spinor bundle. Hitchin [3] proved that the dimension of its kernel, the space of harmonic spinors, only depends on the conformal class of the Riemannian metric, and that it varies with the conformal class. However, the variation is not understood.

Investigating this variation is particularly interesting for manifolds of dimension $4 k$. In that case the Dirac operator interchanges spinors of different chirality and the index of the (half-) Dirac operator $D: V_{+} \rightarrow V_{-}$given by the $\hat{A}$-genus can be non-zero. We say that a conformal structure admits non-trivial harmonic spinors if the dimension of the space of harmonic spinors is larger than $\mid$ index $(D) \mid$. This is equivalent to the existence of harmonic spinors of both chiralities. The natural problem to consider is:

Problem 1. Does there always exist a conformal structure for which there are no non-trivial harmonic spinors? Are such conformal structures generic?

For manifolds of dimension 4, this question is related to the applications of Witten's monopole equations [7].

In this note we address the complex analytic analog of Problem 1. For a complex manifold $X$, the existence of a spin structure is equivalent to the existence of a halfcanonical divisor $\frac{1}{2} K \in H^{2}(X, \mathbb{Z})$. Hodge theory gives canonical isomorphisms between the spaces of harmonic spinors with respect to a Kähler metric and certain sheaf cohomology groups, as follows:

$$
\begin{gathered}
\operatorname{ker}(D)=H^{\text {even }}\left(X, \mathcal{O}\left(\frac{1}{2} K\right)\right), \\
\operatorname{coker}(D)=H^{\text {odd }}\left(X, \mathcal{O}\left(\frac{1}{2} K\right)\right) .
\end{gathered}
$$

Received by the editors December 11, 1994.

1991 Mathematics Subject Classification. Primary 14J99, 53C55, 58D17.

This note was written while the author was an EPSRC Visiting Fellow at the Isaac Newton Institute for Mathematical Sciences in Cambridge.

(c)1996 American Mathematical Society 
This shows that the dimension of the space of harmonic spinors is independent of the choice of Kähler metric compatible with a fixed complex structure. The complex analytic version of Problem 1 is:

Problem 2. If $X$ is a Kählerian compact complex manifold, does there exist a Kähler complex structure for which there are no non-trivial harmonic spinors, i.e. such that

$$
\sum_{i} \operatorname{dim}\left(H^{i}\left(\mathcal{O}\left(\frac{1}{2} K\right)\right)\right)=\left|\chi\left(\mathcal{O}\left(\frac{1}{2} K\right)\right)\right| ?
$$

It is important to keep in mind that, unlike the cohomology of $\mathcal{O}(n K)$ for $n \in$ $\mathbb{Z}$, the cohomology of $\mathcal{O}\left(\frac{1}{2} K\right)$ is not invariant under deformations of the complex structure. There are (non-simply-connected) counterexamples already in complex dimension 1. However, the cohomology is semicontinuous, which implies that if a complex structure exists with no non-trivial harmonic spinors, then a generic complex structure also has this property.

In the direction of Problem 2, Hitchin [3] proved that a simply connected spin Kähler surface which is not of general type or is a complete intersection or a cyclic branched cover of $\mathbb{C} P^{2}$ with smooth branch locus does not have non-trivial harmonic spinors. Hitchin went on to conjecture that for a generic complex structure on a simply connected spin algebraic surface $H^{1}\left(\mathcal{O}\left(\frac{1}{2} K\right)\right)=0$. That this cannot be true as stated follows from the existence of simply connected spin algebraic surfaces for which $\chi\left(\mathcal{O}\left(\frac{1}{2} K\right)\right)<0$ due to Moishezon-Teicher [6], compare [4]. The correct formulation of Hitchin's conjecture is that there should be no non-trivial harmonic spinors for a generic complex structure on a simply connected surface, meaning

$$
H^{1}\left(\mathcal{O}\left(\frac{1}{2} K\right)\right)=0 \quad \text { if } \chi\left(\mathcal{O}\left(\frac{1}{2} K\right)\right) \geq 0
$$

and

$$
H^{0}\left(\mathcal{O}\left(\frac{1}{2} K\right)\right)=H^{2}\left(\mathcal{O}\left(\frac{1}{2} K\right)\right)=0 \quad \text { if } \chi\left(\mathcal{O}\left(\frac{1}{2} K\right)\right) \leq 0 .
$$

In [4], we showed that the Moishezon-Teicher surfaces have spaces of non-trivial harmonic spinors which can be arbitrarily large. However, we do not know whether their complex structures are generic.

We now show that Hitchin's conjecture is false, and that the answer to Problem 2 is negative.

Theorem 1. For each $k>0$ there exist simply connected spin algebraic surfaces $X$ with the following properties:

(i) the $\operatorname{dim}^{i}\left(\mathcal{O}\left(\frac{1}{2} K\right)\right)$ are deformation invariants of $X$, and

(ii) $\sum_{i} \operatorname{dim}\left(H^{i}\left(\mathcal{O}\left(\frac{1}{2} K\right)\right)\right) \geq\left|\chi\left(\mathcal{O}\left(\frac{1}{2} K\right)\right)\right|+k$.

Proof. Examples of such surfaces $X$ include the so-called simple bihyperelliptic surfaces or bidouble covers of $\mathbb{C} P^{1} \times \mathbb{C} P^{1}$ introduced by Catanese [1] and studied further by Catanese [2] and by Manetti [5]. A minimal surface $X$ of general type is a bidouble cover of $\mathbb{C} P^{1} \times \mathbb{C} P^{1}$ of type $(a, b)(m, n)$ if its canonical model is defined in the total space of $\mathcal{O}(a, b) \oplus \mathcal{O}(m, n)$ over $\mathbb{C} P^{1} \times \mathbb{C} P^{1}$ by a pair of equations

$$
z^{2}=f(x, y), \quad w^{2}=g(x, y)
$$


where $f$ and $g$ are bihomogeneous polynomials of bidegrees $(2 a, 2 b)$ and $(2 m, 2 n)$ respectively.

We consider the case where each of $a, b, m, n$ is at least 4 . In this case Catanese [1] showed that the surfaces are simply connected and satisfy

$$
K_{X}=\pi^{*} \mathcal{O}_{\mathbb{C} P^{1} \times \mathbb{C} P^{1}}(n+a-2, m+b-2),
$$

$$
\chi\left(\mathcal{O}_{X}\right)=2(n+a-1)(m+b-1)+2-\frac{1}{2}(m+b)(n+a)+\frac{1}{2}(a-n)(b-m) .
$$

Moreover, if $a \geq \max \{2 n+1, b+2\}$ and $m \geq \max \{2 b+1, n+2\}$, then these surfaces form an irreducible component [2], and even a connected component [5], of the moduli space of surfaces of general type. Thus, all the deformations of a bidouble cover are bidouble covers of the same type.

If $a+n$ and $b+m$ are even, then $K_{X}$ is $2-$ divisible and so $X$ is spin. As it is simply connected, it has a unique spin structure, or half-canonical divisor $\frac{1}{2} K_{X}$. From equations (1) and (2) we obtain

$$
\begin{aligned}
\chi\left(\mathcal{O}\left(\frac{1}{2} K\right)\right) & =\chi\left(\mathcal{O}_{X}\right)-\frac{1}{8} K^{2} \\
& =\frac{1}{2}(n+a)(m+b)+\frac{1}{2}(a-n)(b-m) \\
& =a b+m n>0 .
\end{aligned}
$$

On the other hand, we have

$$
\begin{aligned}
\operatorname{dim} H^{0}\left(\mathcal{O}\left(\frac{1}{2} K\right)\right) & =\operatorname{dim} H^{0}\left(\mathcal{O}_{\mathbb{C} P^{1} \times \mathbb{C} P^{1}}\left(\frac{n+a-2}{2}, \frac{m+b-2}{2}\right)\right) \\
& =\frac{1}{4}(n+a)(m+b) .
\end{aligned}
$$

By Serre duality, we have $\operatorname{dim} H^{2}\left(\mathcal{O}\left(\frac{1}{2} K\right)\right)=\operatorname{dim} H^{0}\left(\mathcal{O}\left(\frac{1}{2} K\right)\right)$. We conclude that

$$
\begin{aligned}
\sum_{i} \operatorname{dim}\left(H^{i}\left(\mathcal{O}\left(\frac{1}{2} K\right)\right)\right)-\left|\chi\left(\mathcal{O}\left(\frac{1}{2} K\right)\right)\right| & =2 \operatorname{dim}^{1}\left(\mathcal{O}\left(\frac{1}{2} K\right)\right) \\
& =2\left(-\chi\left(\mathcal{O}\left(\frac{1}{2} K\right)\right)+2 \operatorname{dim}\left(H^{0}\left(\mathcal{O}\left(\frac{1}{2} K\right)\right)\right)\right) \\
& =(a-n)(m-b)>0 .
\end{aligned}
$$

By choosing $a, b, m, n$ appropriately, we can make this arbitrarily large.

\section{ACKNOWLEDGEMENT}

The author wishes to thank F. Catanese for some helpful comments.

\section{REFERENCES}

1. F. Catanese, On the moduli spaces of surfaces of general type, J. Diff. Geo. 19 (1984), 483-515. MR 86h:14031

2. F. Catanese, Automorphisms of rational double points and moduli spaces of surfaces of general type, Comp. Math. 61 (1987), 81-102. MR 88c:14056

3. N. Hitchin, Harmonic Spinors, Adv. in Math. 14 (1974), 1-55. MR 50:11332

4. D. Kotschick, Non-trivial harmonic spinors on certain algebraic surfaces, in "Einstein metrics and Yang-Mills connections", ed. T. Mabuchi and S. Mukai, Marcel Dekker, New York, Basel, Hong Kong 1993. MR 94d:58138 
5. M. Manetti, On some components of moduli space of surfaces of general type, Comp. Math. 92 (1994), 285-297. MR 95h:14028

6. B. Moishezon and M. Teicher, Simply-connected algebraic surfaces of positive index, Invent. Math. 89 (1987), 601-643. MR 88f: 14037

7. E. Witten, Monopoles and 4-manifolds, Math. Research Letters 1 (1994), 769-796. CMP 95:05

Mathematisches Institut, Universität Basel, Rheinsprung 21, 4051 Basel, SwitzerLAND

E-mail address: dieter@math.unibas.ch 